PROC. OF JSCE,

No. 214, JUNE 1973

\title{
OUT-OF-PLANE BEHAVIOR OF CIRCULAR ARCHES UNDER SIDE LOADINGS
}

\author{
By Tetsuya YABUKI* and Shigeru KURANISHI ${ }^{* *}$
}

\begin{abstract}
SYNOPSIS
This paper presents out-of-plane behavior of circular arches subjected to lateral loads. The arches are analysed by dividing it in three components (two arch ribs and a lateral bracing system) and by solving the elastic differential equations for them with the aid of the Laplace transformation method. In relation to interaction of flexural rigidity of cross beam, torsional rigidity of arch ribs and warping rigidity as a whole cross section of the arch, the effect of biaxial flexural rigidity of individual arch ribs on their out-of-plane behavior is chiefly investigated. The effect of end-supporting conditions of the arches is also discussed.
\end{abstract}

\section{INTRODUCTION}

Several investigations dealing with the out-ofplane behavior of arches have already been conducted. S. Okamoto ${ }^{1)}$ studied the out-of-plane behavior of circular arches neglecting the warping rigidity. P. Donald and W. Godden ${ }^{2}$ gave a solution dealing with the out-of-plane behavior of a single arch rib by a numerical forward integration method. One of the authors ${ }^{3}$ already presented some results to the out-of-plane behavior of this type of arch subjected to a uniformly distributed lateral load.

In the previous paper, the effect of the biaxial flexural rigidity of individual arch ribs is neglected. Even if two arch ribs have themselves high torsional rigidity, an arch composed of single lateral bracing and cross beams with low flexural rigidity or connected with pins, is not able to be expected its torsional rigidity to the out-of-plane,

* Research Assistant of Civil Engineering, Tohoku University.

** Dr. Engg., Professor of Civil Engineering, Tohoku University. i.e., the whole cross section of the arch is twisted by the difference between the vertical displacements of two arch ribs and the flexural rigidity of the arch ribs has no effect on the torsional rigidity of the ribs. The out-of-plane deformation of arches, however, occurs in a coupled action of its bending and twisting by reason of its curvature. Therefore, the torsional rigidity of the individual arch ribs is subjected to the influence of lateral bending.

From this point of view, the effect of the interaction between the torsional rigidity and the outof-plane flexural rigidity of arch ribs on the bending moment, the torsional moment and the lateral deformation of the ribs are calculated and examined, respectively.

The analysis adopted is performed by representing the arch by circularly curved beams connected each other by a rigid lateral system and flexural cross beams and by solving the elastic differential equations for them with the aid of the Laplace transformation method. The analysis in this paper is confined in the 1st order elastic one, so that the influence of the axial forces and in-plane loadings on the out-of-plane displacements of the ribs is disregarded here.

\section{BASIC EQUATIONS}

\section{(1) Formation of Equations}

The equations of the elastic curve of an arch can be set up respectively for an arch axis and two arch rib axes, where the arch axis is expressed by a curved line joining the middle points of lines that connect the centroidal points of both cross sections of the arch ribs, and the arch rib axis is a centroidal axis of its cross section, respectively. These three basic differential equations are combined by the relation of the deformation and flexural rigidity of cross beams and expressed by Eqs. (2-12), (2-13) and (2-14). 


\section{(2) Assumptions}

In this analysis, the basic equations are derived from the following assumptions:

1) Arch axis and two arch rib axes are circular.

2) Cross sections of arch ribs are constant along the entire arc's length and double symmetric, i.e., the elastic center and the shear center of a cross section of arch rib coincide with each other.

3) The effect of shearing deformation of the lateral bracing is neglected.

4) Cross beams connected rigidly with two arch ribs are distributed uniformly over the whole length of the arch.

5) Lateral loads act on the arch axis.

6) The analysis bases on the 1st order elastic one.

7) The influence of axial forces and in-plane loadings on the out-of-plane displacements of the ribs is disregarded.

8) The warping rigidity of individual arch ribs is neglected.

\section{(3) Equations for Arch Rib Axis}

An arch rib is represented by its axis. Fig. 1 shows the coordinates system and the displacements of its axis. The reference axes pass through the shear center of a cross section of the arch rib; the $X$-axis is along the arch rib

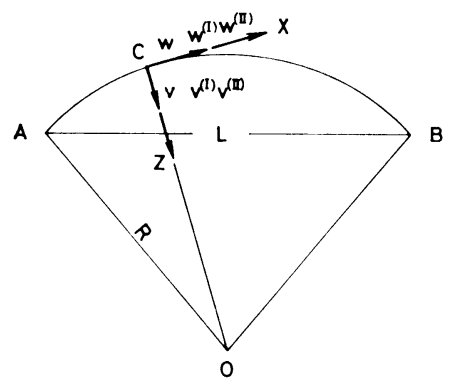

(a) $X-Z$ plane

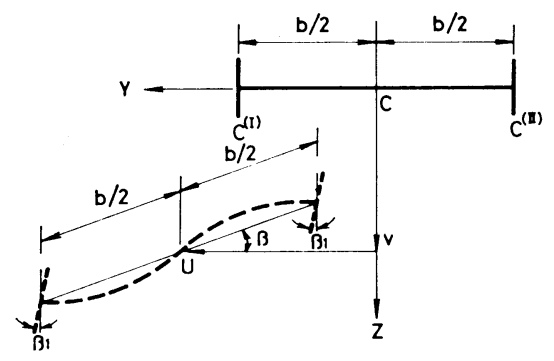

(b) $Y-Z$ plane

Fig. 1 Coordinate system and displacement. axis, the $Y$-axis is the horizontal axis, and $Z$ axis is the radial axis.

In Fig. $1, u^{(\mathrm{I})}, v^{(\mathrm{I})}$ and $w^{(\mathrm{I})}$ are deflection components of the left side rib which are positive in the direction of a positive $Y, Z$ and $X$-coordinate increment, respectively. $\beta^{(1)}$ is the twist angle of the rib and is taken positive, when the counterclockwise rotation vector points in the direction of $X$-coordinate. The deformation components of the right side rib are denoted by $u^{(\mathrm{II})}, v^{(\mathrm{II})}, w^{(\mathrm{II})}$ and $\beta^{(\mathrm{II})}$ respectively. But, it is assumed here that $u^{(\mathrm{I})}=u^{(\mathrm{II})}=u$, because of negligibly small shortening of cross beams. By the assumption (2), it is expressed that $\beta^{(\mathrm{I})}=\beta^{(\mathrm{II})}=\beta_{1}$.

The equation of the elastic curve of the arch rib loaded by lateral forces is obtained by expressing the curvature and twist components in terms of the deflection $u$ and the twist angle $\beta_{1}$. The torsional moment $M_{t}^{(\mathrm{I}) \text {,(II) }}$ and the bending moment about $Z$-axis $M_{z}^{(\mathrm{I}) \text {,(II) }}$ acting on the individual arch rib axes, will be written respectively as

$$
\begin{aligned}
& M_{t}^{(\mathrm{I})}=M_{t}^{(\mathrm{II})}=M_{t}=\frac{G J_{T}}{R}\left(\frac{d \beta_{1}}{d \phi}-\frac{1}{R} \frac{d u}{d \phi}\right) \\
& M_{z}^{(\mathrm{I})}=M_{z}^{(\mathrm{II})}=M_{z}=\frac{E J_{z}}{R}\left(\frac{1}{R} \frac{d^{2} u}{d \phi^{2}}+\beta_{1}\right)
\end{aligned}
$$

The bending moments $M_{y}^{(1),(I I)}$ about the $Y$ axis of the two arch ribs will be written as

$$
\begin{gathered}
M_{y}^{(\mathrm{I})=}-\frac{E J_{y}}{R}\left\{\frac{d^{2} v}{d \phi^{2}}+v+\frac{b}{2}\left(\frac{d^{2} \beta}{d \phi^{2}}-\frac{1}{R} \frac{d^{2} u}{d \phi^{2}}\right)\right\} \\
\ldots \ldots \ldots \ldots \ldots \ldots \ldots \ldots \ldots \ldots \ldots \ldots \ldots \ldots \ldots \ldots \ldots \ldots \ldots \\
M_{y}^{(\mathrm{II})}=-\frac{E J_{y}}{R}\left\{\frac{d^{2} v}{d \phi^{2}}+v-\frac{b}{2}\left(\frac{d^{2} \beta}{d \phi^{2}}-\frac{1}{R} \frac{d^{2} u}{d \phi^{2}}\right)\right\}
\end{gathered}
$$

\section{(4) Equations for Arch Axis}

The torsional moment $M_{T}$ and the bending moment about $Z$-axis $M_{Z}$ acting on a whole cross section of the arch, are expressed as follows:

$$
\begin{aligned}
& M_{T}=2 M_{t}+\frac{b}{2}\left(Q^{(\mathrm{II})}-Q^{(\mathrm{I})}\right) \cdots \cdots \\
& M_{Z}=2 M_{z}-\frac{E J_{Z}}{R}\left(\frac{1}{R} \frac{d^{2} u}{d \phi^{2}}+\beta\right)
\end{aligned}
$$

From Eqs. (2-3.a) and (2-3.b), we get

$$
Q^{(\mathrm{II})}-Q^{(\mathrm{I})}=\frac{b E J_{y}}{R^{3}}\left(\frac{d^{3} \beta}{d \phi^{3}}-\frac{1}{R} \frac{d^{3} u}{d \phi^{3}}\right)
$$

Obviously the individual flexural rigidity of arch ribs about horizontal axis works as the warping rigidity to a whole cross section. Substituting 
Eqs. (2-1), (2-2) and (2-6) into Eqs. (2-4) and (2-5), $M_{T}$ and $M_{Z}$ are given as follows:

$$
\begin{aligned}
M_{T^{\prime}}= & \frac{2 G J_{T}}{R}\left(\frac{d \beta_{1}}{d \phi}-\frac{1}{R} \frac{d u}{d \phi}\right) \\
& -\frac{b^{2} E J_{v}}{2 R^{3}}\left(\frac{d^{3} \beta}{d \phi^{3}}-\frac{1}{R} \frac{d^{3} u}{d \phi^{3}}\right) \\
M_{Z}= & -\frac{2 E J_{z}}{R}\left(\frac{1}{R} \frac{d^{2} u}{d \phi^{2}}+\beta_{1}\right) \\
& -\frac{E J_{Z}}{R}\left(\frac{1}{R} \frac{d^{2} u}{d \phi^{2}}+\beta\right) \ldots \ldots .
\end{aligned}
$$

\section{(5) Deformation of Cross Beam}

The bending moment acting on the ends of a cross beam is given (see Fig. 1 (b))

$$
M_{q}=\frac{6 E J_{q}}{b}\left(\beta-\beta_{1}\right)
$$

From the assumption (4) for cross beams, the end moment per unit length $m_{q}$ is

$$
m_{q}=\frac{6 E J_{q}}{b \lambda}\left(\beta-\beta_{1}\right)
$$

\section{(6) Differential Equations}

Let us consider the equilibrium of an infinitesimal length element of the arch axis. Sectional forces acting on the cross section of the element are the transverse shear $Q$, the bending moment $M_{Z}$ about the $Z$-axis and the torsional moment $M_{T}$. A uniformly distributed load $q$ and torque $m_{T}$ are acting on the element in addition to them. The equilibrium equations for deformation of the element with respect to the $X-, Z$ and $Y$-axis are

$$
\begin{aligned}
& \frac{1}{R} \frac{d M_{Z}}{d \phi}-Q-\frac{M_{T}}{R}=0 \\
& \frac{d M_{T}}{d \phi}+M_{Z}+R m_{T}=0 \\
& q-\frac{1}{R} \frac{d Q}{d \phi}=0 \ldots \ldots \ldots \ldots . . .
\end{aligned}
$$

Substituting the relation of Eqs. (2-7) and (2-8) into Eq. (2-11) after eliminating $Q$, the following basic differential equations are obtained, which include the effect of the biaxial flexural rigidity of the arch ribs:

$$
\begin{aligned}
& 2 G J_{T}\left(\beta_{1}^{\prime \prime}-\frac{u^{\prime \prime}}{R}\right)-\frac{b^{2} E J_{y}}{2 R^{2}}\left(\beta^{\mathrm{IV}}-\frac{u^{\mathrm{IV}}}{R}\right) \\
&+R M_{Z}=0 \cdots \cdots \cdots \cdots \cdots(2-12) \\
& 2 E J_{z}\left(\beta_{1}+\frac{u^{\prime \prime}}{R}\right)+E J_{Z}\left(\beta+\frac{u^{\prime \prime}}{R}\right)+R M_{Z}=0
\end{aligned}
$$

in which ' means differentiation with respect to $\phi$. The end moment $m_{q}$ of the cross beams as shown in Eq. (2-10) acts on the individual arch ribs as external distributed torque. Therefore, by substituting Eqs. (2-1), (2-2) and (2-10) into Eq. (2-11.b), the equation connecting deformation and force of the two arch rib axes and arch axis is obtained as follows:

$$
\begin{gathered}
G J_{T}\left(\beta_{1}{ }^{\prime \prime}-\frac{u^{\prime \prime}}{R}\right)-E J_{z}\left(\beta_{1}+\frac{u^{\prime \prime}}{R}\right) \\
+\frac{6 R^{2} E J_{q}}{b \lambda}\left(\beta-\beta_{1}\right)=0 \quad \ldots \ldots \ldots .
\end{gathered}
$$

Eqs. (2-12), (2-13) and (2-14) present the basic elastic equations. If the flexural rigidity of cross beams is infinitely high and $J_{z}=0$, Eqs. (2-12) and (2-13) are rewritten as follows:

$$
\begin{aligned}
& 2 G J_{T}\left(\beta^{\prime \prime}-\frac{u^{\prime \prime}}{R}\right)-\frac{b^{2} E J_{y}}{2 R^{2}}\left(\beta^{\mathrm{IV}}-\frac{u^{\mathrm{IV}}}{R}\right) \\
& +R M_{Z}=0 \\
& E J_{Z}\left(\beta+\frac{u^{\prime \prime}}{R}\right)+R M_{Z}=0
\end{aligned}
$$

These are the basic differential equations for the case in which the arch is represented by an equivalent circular beam with undeformable cross section.

\section{(7) Loading}

The positive direction of a concentrated load $P$ and a uniformly distributed load $q$ are shown

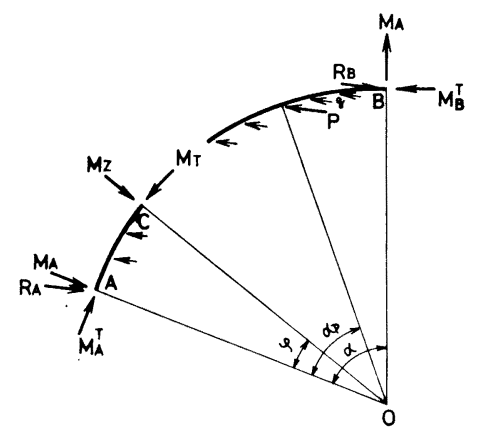

Fig. 2 Loading condition.

in Fig. 2. $M_{T}$ and $M_{Z}$ are moment components acting on a cross section of the arch axis and taken positive when the arc $\overparen{B C}$ is twisted counterclockwise and deflects to the positive direction of the loads. $M_{Z}$ and $M_{T}$ are obtained as follows:

$$
\begin{aligned}
M_{Z}= & M_{1} \sin \phi+M_{2} \cos \phi \\
& +R P \delta(\varphi) \sin \left(\varphi-\alpha_{P}\right)-q R^{2} \ldots \ldots(3-1 . \mathrm{a}) \\
M_{1}= & \frac{\cos \alpha}{\sin \alpha} M_{A}-\frac{1}{\sin \alpha} M_{B}+\frac{\sin \left(\alpha-\alpha_{P}\right)}{\sin \alpha} R P \\
& +\frac{1-\cos \alpha}{\sin \alpha} R^{2} q \ldots \ldots \ldots \ldots \ldots \ldots(3-1 . \mathrm{b})
\end{aligned}
$$




$$
\begin{aligned}
& M_{2}=-M_{A}+R^{2} q \\
& M_{T}=M_{A}{ }^{T} \cos \phi+M_{A} \sin \phi-R R_{A}(1-\cos \phi) \\
& +R P\left[1-\cos \left(\phi-\alpha_{P}\right)\right] \delta(\phi)+R^{2} q(\phi-\sin \phi) \\
& \delta(\phi)=\left\{1:\left(\phi \geqq \alpha_{P}\right), 0:\left(\phi<\alpha_{P}\right)\right\}
\end{aligned}
$$

\section{(8) Boundary Conditions}

The arch studied in this paper is composed of two arch ribs connected each other by cross beams and lateral bracing. For the arch like this type, two kinds of supporting conditions will be considered. Namely one is that the cross section of the arch is fixed perfectly at the end supports (support type A) and another is that lateral deflection is fixed, however, the plane of the end cross section is free to warp (support type B). In this study, the following boundary conditions are taken up at the end supports.

Expressed mathematically, they are respectively

a) support type $\mathrm{A}$

$$
\left.\begin{array}{ll}
u(0)=u(\alpha)=0, & u^{\prime}(0)=u^{\prime}(\alpha)=0 \\
\beta(0)=\beta(\alpha)=0, & \beta_{1}(0)=\beta_{1}(\alpha)=0 \\
\beta_{1}{ }^{\prime}(0)=\beta_{1}{ }^{\prime}(\alpha)=0 &
\end{array}\right\}
$$

b) support type $B$

$$
\left.\begin{array}{l}
u(0)=u(\alpha)=0, \quad u^{\prime}(0)=u^{\prime}(\alpha)=0 \\
\beta(0)=\beta(\alpha)=0, \quad \beta_{1}(0)=\beta_{1}(\alpha)=0 \\
B_{W}(0)=B_{W}(\alpha)=0
\end{array}\right\}
$$

in which

$$
B_{W}(\phi)=\frac{E J_{Z}}{q R^{3}}\left\{\beta^{\prime \prime}(\phi)-\frac{1}{R} u^{\prime \prime}(\phi)\right\}
$$

Therefore, the warping normal stress $\sigma_{W}$ is expressed as follows:

$$
\sigma_{W}=\frac{e b R q}{2 J_{Z}} B_{W}(\phi)
$$

in which $e$ is the distance between neutral axis and the top or bottom surface of arch rib.

\section{SOLUTIONS}

\section{(1) Solution of Basic Differential Equations}

The basic simultaneous differential equations Eqs. (2-12), (2-13), (2-14) and the bending moment equation (3-1) will be solved by using the Laplace transformation method.

From Eqs. (2-12), (2-13), (2-14) and (3-1), the equations are rewritten as follows:

$$
\begin{aligned}
& C_{T}\left(\beta_{1}^{\prime \prime}-\frac{u^{\prime \prime}}{R}\right)-C_{b d}\left(\beta^{\mathrm{IV}}-\frac{u^{\mathrm{IV}}}{R}\right)+R M_{Z}=0 \\
& C_{j}\left(\beta_{1}+\frac{u^{\prime \prime}}{R}\right)+C_{J}\left(\beta+\frac{u^{\prime \prime}}{R}\right)+R M_{Z}=0 \\
& C_{T}\left(\beta_{1}^{\prime \prime}-\frac{u^{\prime \prime}}{R}\right)-C_{j}\left(\beta_{1}+\frac{u^{\prime \prime}}{R}\right)+C_{q}\left(\beta-\beta_{1}\right)=0 \\
& M_{1} \sin \phi+M_{2} \cos \phi+R P \delta(\phi) \sin \left(\phi-\alpha_{P}\right) \\
& -q R^{2}-M_{Z}=0 \\
& C_{b d}=b^{2} E J_{y} / 2 R^{2} \text { and } C_{q}=12 R^{2} E J_{q} / b \lambda
\end{aligned}
$$

By applying the Laplace transformation on Eqs. (5-1.a) (5-1.d) and by considering the boundary conditions at the one end support $(\phi=0)$, as shown in Eqs. (4-1) and (4-2), the solution for the deflection is obtained as follows:

$$
\begin{aligned}
& \beta_{1}(\phi)=B C_{1} \cdot X_{\beta 1}^{1}(\phi)+B C_{2} \cdot X_{\beta 1}^{2}(\phi)+B C_{3} \cdot X_{\beta 1}^{3}(\phi) \\
& +\bar{M}_{A} \cdot Y_{\beta 1}^{1}(\phi)+\bar{M}_{B} \cdot Y_{\beta 1}^{2}(\phi)+\bar{P} \cdot Y_{\beta}^{3}(\phi) \\
& +\bar{Q} \cdot Y_{\beta}^{4}(\phi) \\
& \beta(\phi)=B C_{1} \cdot X_{\beta}{ }^{1}(\phi)+B C_{2} \cdot X_{\beta}{ }^{2}(\phi)+B C_{3} \cdot X_{\beta}{ }^{3}(\phi) \\
& +\bar{M}_{A} \cdot Y_{\beta}{ }^{1}(\phi)+\bar{M}_{B} \cdot Y_{\beta}{ }^{2}(\phi)+\bar{P} \cdot Y_{\beta}{ }^{3}(\phi) \\
& +\bar{Q} \cdot Y_{\beta}{ }^{4}(\phi) \\
& \frac{u(\phi)}{R}=B C_{1} \cdot X_{u}{ }^{1}(\phi)+B C_{2} \cdot X_{u^{2}}(\phi)+B C_{3} \cdot X_{u^{3}}(\phi) \\
& +\bar{M}_{A} \cdot Y_{u^{1}}(\phi)+\bar{M}_{B} \cdot Y_{u^{2}}(\phi)+\bar{P} \cdot Y_{u^{3}}(\phi) \\
& +\bar{Q} \cdot Y_{u}{ }^{4}(\phi)
\end{aligned}
$$

in which $\bar{M}_{A}=R M_{A} / E J_{Z}, \bar{M}_{B}=R M_{B} / E J_{Z}, \quad \bar{P}=$ $P R^{2} / E J_{Z}, \bar{Q}=q R^{3} / E J_{Z} . \quad B C_{1}, B C_{2}$ and $B C_{3}$ are initial constants. Expressed mathematically, they are respectively:

a) support type $\mathrm{A}$

$$
\begin{aligned}
B C_{1}=\beta^{\prime}(0), \quad B C_{2} & =\beta^{\prime \prime}(0)-\frac{u^{\prime \prime}(0)}{R}, \\
B C_{3} & =\beta^{\prime \prime \prime}(0)-\frac{u^{\prime \prime \prime}(0)}{R} .
\end{aligned}
$$

b) support type $B$

$$
\begin{aligned}
B C_{1}=\beta^{\prime}(0), \quad B C_{2} & =\beta_{1}^{\prime}(0), \\
B C_{3} & =\beta^{\prime \prime \prime}(0)-\frac{u^{\prime \prime \prime}(0)}{R} .
\end{aligned}
$$

Coefficients of the initial constants and nondimensional external forces in Eqs. (5-2.a) (5-2.c), are shown in Appendix I.

\section{(2) Determination of Initial Constants}

Expressing the initial constants $B C_{i}(i=1,2,3)$ by the terms of nondimensional external forces $\bar{M}_{A}, \bar{M}_{B}, \bar{P}$ and $\bar{Q}$, we get 


$$
\begin{gathered}
{\left[\begin{array}{l}
B C_{1} \\
B C_{2} \\
B C_{3}
\end{array}\right]=\bar{M}_{A}\left[\begin{array}{l}
B C_{11} \\
B C_{21} \\
B C_{31}
\end{array}\right]+\bar{M}_{B}\left[\begin{array}{l}
B C_{12} \\
B C_{22} \\
B C_{33}
\end{array}\right]+\bar{P}\left[\begin{array}{l}
B C_{13} \\
B C_{23} \\
B C_{33}
\end{array}\right]} \\
+\bar{Q}\left[\begin{array}{l}
B C_{14} \\
B C_{24} \\
B C_{34}
\end{array}\right] \ldots \ldots \ldots(5-4)
\end{gathered}
$$

in which $B C_{i k}(i=1,2,3, k=1,2,3,4)$ mean each of component terms for the external forces. By substituting the boundary conditions $\beta(\alpha)=u(\alpha)=$ $u^{\prime}(\alpha)=0$, which are common to two kinds of the supporting conditions, the following equation is obtained:

$$
\begin{gathered}
{\left[\begin{array}{lll}
E_{11} & E_{12} & E_{13} \\
E_{21} & E_{22} & E_{23} \\
E_{31} & E_{32} & E_{33}
\end{array}\right] \cdot\left[\begin{array}{l}
B C_{1} \\
B C_{2} \\
B C_{3}
\end{array}\right]=\bar{M}_{A}\left[\begin{array}{l}
D_{11} \\
D_{21} \\
D_{31}
\end{array}\right]} \\
+\bar{M}_{B}\left[\begin{array}{l}
D_{12} \\
D_{22} \\
D_{32}
\end{array}\right]+\bar{P}\left[\begin{array}{c}
D_{13} \\
D_{23} \\
D_{33}
\end{array}\right]+\bar{Q}\left[\begin{array}{c}
D_{14} \\
D_{24} \\
D_{34}
\end{array}\right] \cdots(5-5 . \mathrm{a})
\end{gathered}
$$

Where $E_{j i}$ and $D_{j k}$ are shown as follows:

$$
\left.\begin{array}{l}
E_{1 i}=X_{\beta}^{i}(\alpha), \quad E_{2 i}=X_{u^{i}}(\alpha), \quad E_{3 i}=X_{u^{i \prime}}(\alpha), \\
D_{1 k}=-Y_{\beta}^{k}(\alpha), \quad D_{2 k}=-Y_{u^{k}}(\alpha), \\
D_{3 k}=-Y_{u^{k \prime}}(\alpha) \quad(i=1,2,3, \quad k=1,2,3,4)
\end{array}\right\}
$$

By substituting Eq. (5-4) into Eqs. (5-5.a) and (5-5.b) and collecting like terms, each of component terms for the external forces are expressed as

$$
\begin{aligned}
& {\left[\begin{array}{llll}
B C_{11} & B C_{12} & B C_{13} & B C_{14} \\
B C_{21} & B C_{22} & B C_{23} & B C_{24} \\
B C_{31} & B C_{32} & B C_{33} & B C_{34}
\end{array}\right] } \\
= & {\left[\begin{array}{lll}
E_{11} & E_{12} & E_{13} \\
E_{21} & E_{22} & E_{23} \\
E_{31} & E_{32} & E_{33}
\end{array}\right]^{-1} \cdot\left[\begin{array}{llll}
D_{11} & D_{12} & D_{13} & D_{14} \\
D_{21} & D_{22} & D_{23} & D_{24} \\
D_{31} & D_{32} & D_{33} & D_{34}
\end{array}\right] }
\end{aligned}
$$

By substituting Eq. (5-6) into Eqs. (5-2.a) (5-2.c), the lateral displacement and the twist angles are expressed also by the terms of the external forces:

$$
\begin{aligned}
\beta_{1}(\phi)= & \bar{M}_{A} \cdot Z_{\beta}{ }_{1}^{1}(\phi)+\bar{M}_{B} \cdot Z_{\beta 1}{ }^{2}(\phi) \\
& +\bar{P} \cdot Z_{\beta}{ }^{3}(\phi)+\bar{Q} \cdot Z_{\beta}{ }^{4}(\phi) \cdots \\
\beta(\phi)= & \bar{M}_{A} \cdot Z_{\beta}{ }^{1}(\phi)+\bar{M}_{B} \cdot Z_{\beta}{ }^{2}(\phi) \\
& +\bar{P} \cdot Z_{\beta}{ }^{3}(\phi)+\bar{Q} \cdot Z_{\beta}{ }^{4}(\phi) \cdots \\
\frac{u(\phi)}{R}= & \bar{M}_{A} \cdot Z_{u}{ }^{1}(\phi)+\bar{M}_{B} \cdot Z_{u^{2}(\phi)} \\
& +\bar{P} \cdot Z_{u}{ }^{3}(\phi)+\bar{Q} \cdot Z_{u^{4}}(\phi) \cdots
\end{aligned}
$$

in which coefficients of the external forces are as follows:

$$
\begin{gathered}
Z_{\beta}^{k}(\phi)=\sum_{i=1}^{3} X_{\beta 1}^{i}(\phi) B C_{i k}+Y_{\beta 1}^{k}(\phi) \cdots \cdots(5-8 . \mathrm{a}) \\
Z_{\beta}^{k}(\phi)=\sum_{i=1}^{3} X_{\beta}{ }^{i}(\phi) B C_{i k}+Y_{\beta}{ }^{k}(\phi) \cdots \cdots(5-8 . \mathrm{b}) \\
Z_{u^{k}}(\phi)=\sum_{i=1}^{3} X_{u}{ }^{i}(\phi) B C_{i k}+Y_{u}{ }^{k}(\phi) \cdots \cdots(5-8 . \mathrm{c}) \\
(k=1,2,3,4)
\end{gathered}
$$

\section{(3) Determination of End Moments}

The boundary conditions left unused in Eqs. (4-1) and (4-2) give the end-bending moments. They are, respectively, for support type A:

$$
\beta_{1}(\alpha)=0, \quad \beta_{1}^{\prime}(\alpha)=0
$$

for support type $B$ :

$$
\beta_{1}(\alpha)=0, \quad \beta^{\prime \prime}(\alpha)-u^{\prime \prime}(\alpha) / R=0
$$

The same procedure is applied to Eq. (5-9.b). Substituting the relations $\beta(\alpha)=\beta_{1}(\alpha)=0$ into Eqs. (2-8) and (3-1) leads the following equation:

$$
M_{Z}(\alpha)=-M_{B}=-\left(C_{j}+C_{J}\right) \frac{u^{\prime \prime}(\alpha)}{R} \cdots \cdots(5-9 . c)
$$

From Eqs. (5-9.b) and (5-9.c), therefore, the boundary conditions for support type $B$ are rewritten as follows, which are used to determine the endbending moment:

$$
\beta^{\prime \prime}(\alpha)=\frac{u^{\prime \prime}(\alpha)}{R}=\left(1+\frac{C_{j}}{C_{J}}\right) \bar{M}_{B}
$$

The end-bending moments $\bar{M}_{A}$ and $\bar{M}_{B}$, for the cases of support type $A$ and type $B$, are determined by substituting Eqs. (5-9.a) and (5-9.d) into Eqs. (5-7.a) (5-7.c):

$$
\left[\begin{array}{l}
\bar{M}_{A} \\
\bar{M}_{B}
\end{array}\right]=\left[\begin{array}{ll}
G_{11} & G_{12} \\
G_{21} & G_{22}
\end{array}\right]^{-1} \cdot\left[\begin{array}{ll}
F_{11} & F_{12} \\
F_{21} & F_{22}
\end{array}\right]\left[\begin{array}{c}
\bar{P} \\
\bar{Q}
\end{array}\right]
$$

in which the each term in Eq. (5-10) is shown as follows:

for type $A$

$$
\begin{aligned}
& G_{11}=Z_{\beta}{ }^{1}(\alpha), \quad G_{12}=Z_{\beta}^{2}(\alpha), \\
& G_{21}=Z_{\beta_{1}^{1}}(\alpha), \quad G_{22}=Z_{\beta 1}{ }^{2}(\alpha) \\
& F_{11}=-Z_{\beta 1}^{3}(\alpha), \quad F_{12}=-Z_{\beta 1}^{4}(\alpha), \\
& F_{21}=-Z_{\beta 1}^{3 \prime}(\alpha), \quad F_{22}=-Z_{\beta 1}{ }^{4 \prime}(\alpha)
\end{aligned}
$$

for type $B$

$$
\begin{aligned}
& G_{11}=Z_{\beta}{ }^{1 \prime}(\alpha), \quad G_{12}=Z_{\beta}{ }^{2 \prime}(\alpha)-\left(1+C_{j} / C_{J}\right) \\
& G_{21}=Z_{u^{1 \prime}}(\alpha), \quad G_{22}=Z_{u^{2 \prime}}(\alpha)-\left(1+C_{j} / C_{J}\right) \\
& F_{11}=-Z_{\beta}{ }^{3 \prime \prime}(\alpha), \quad F_{12}=-Z_{\beta}{ }^{4 \prime}(\alpha), \\
& F_{21}=-Z_{u^{3 \prime \prime}}(\alpha), \quad F_{22}=-Z_{u^{4 \prime \prime}}(\alpha)
\end{aligned}
$$

The end-torsional moment $M_{A}{ }^{T}$ is obtained by substituting Eqs. (4-1), (4-2), (5-3.a) and (5-3.b) into Eq. (2-7): 
$\begin{array}{ll}\text { for type A } & \bar{M}_{A}{ }^{T}=-\frac{C_{b d}}{C_{J}} B C_{3} \cdots(5-11 . \mathrm{a}) \\ \text { for type B } & \bar{M}_{A}{ }^{T}=\frac{C_{T}}{C_{J}} B C_{2}-\frac{C_{b d}}{C_{J}} B C_{3}\end{array}$

in which $\bar{M}_{A}^{T}=R M_{A}^{T} / E J_{Z}$

\section{(4) Computed Results}

In order to evaluate numerically the effect of the biaxial flexural rigidity of the arch ribs on the out-of-plane behavior, the following various parameters are used, in relation to the influence of torsional rigidity of the ribs and of flexural rigidity of cross beams:

$$
\begin{aligned}
\alpha, \quad l=\frac{2 G J_{T}}{E J_{Z}}, \quad m=\frac{J_{z}}{J_{Z}}, \quad n & =\frac{J_{Z} b \lambda}{J_{q} L^{2}}, \\
\rho & =\frac{L}{\sqrt{J_{y} / A}}
\end{aligned}
$$

in which $A$ is the cross sectional area of an arch rib. The limits of parameters used in this calculation are, respectively,

$$
\begin{array}{ll}
0.8 \leqslant \alpha \leqslant 2.0, & 0.1 \leqslant l \leqslant 1.0, \quad 10^{-3} \leqslant m \leqslant 10^{-0.5} \\
10 \leqslant n \leqslant 10^{3}, & 150 \leqslant \rho \leqslant 300
\end{array}
$$

and the lateral loads are $q L^{3} / E J_{Z}=1 / 100$ and $P L^{2} / E J_{Z}=0$. For some cases, the calculation of the matrix determining initial constants (as given in Chapter 3) led the results of low accuracy, by reason of the so-called "ill-conditioning of coefficient's matrix". It occured at the transient range of the boundary from Eqs. (2-12), (2-13) to Eqs. $\left(2-12^{\prime}\right),\left(2-13^{\prime}\right)$. The calculation for the case when the arch is represented by an equivalent circular beam, is carried out using the same parameter and the same dimension except $J_{z}=0$, $J_{q}=\infty$. For the case of support type A, however, even the solution for an equivalent circular beam was not able to be obtained with high accuracy

\begin{tabular}{|c|c|c|c|c|c|c|c|c|c|c|c|c|c|c|}
\hline Result & \multicolumn{2}{|c|}{$\beta(\alpha / 2)$} & \multicolumn{2}{|c|}{$\beta_{1}(\alpha / 2)$} & \multicolumn{2}{|c|}{$\beta_{1}(\alpha / 2) / \beta(\alpha / 2)$} & \multicolumn{2}{|c|}{$u(\alpha / 2) / L$} & \multicolumn{2}{|c|}{$M_{A} /\left(q L^{2}\right)$} & \multicolumn{2}{|c|}{$M_{A} T /\left(q L^{2}\right)$} & \multicolumn{2}{|c|}{$B_{W}$} \\
\hline$l$ & I.C. & L.I. & D.C. & D.C. & D.C. & D.C. & I.C. & I.C. & D.C. & D.C. & I.C. & D.C. & D.C. & D.C. \\
\hline$m$ & D.C. & D.C. & D.C. & D.C. & D.C. & D.C. & D.C. & D.C. & D.C. & D.C. & D.C. & D.C. & $\mathrm{C}, \mathrm{P}$. & D.C. \\
\hline$n$ & C.P. & I.C. & D.C. & D.C. & D.C. & D.C. & C.P. & I.C. & L.I. & L.I. & I.C. & I.C. & I.C. & I.C. \\
\hline$\rho$ & I.C. & I.C. & I.C. & I.C. & D.C. & D.C. & I.C. & I.C. & L.I. & D.C. & L.I. & D.C. & I.C. & I.C. \\
\hline$\alpha$ & C.P. & I.C. & C.P. & I.C. & C.P. & I.C. & C.P. & I.C. & I.C. & I.C. & I.C. & I.C. & I.C. & I.C. \\
\hline
\end{tabular}
within the above mentioned limits of the parameters. The calculation results are listed in

Table 1

In the left (right) side column listing the result tendency, the case of support type A (B) is shown.

Table 1, where we can find some general tendencies. Remarkable characteristics are shown in the following paragraph. (In this Table, "I. C." and "D. C." denote, respectively, that as each parameter increases, the result "increases" and "decreases". Furthermore, "C. P." means that the result is extremely "complicate" to grasp a tendency for the change of the parameter, and "L. I." means that the result have a "less influence" on the change of the parameter.)

a) Effect of Rigidity Ration $m$ and $n$

Figs. 3 and 4 show the relationship between the twist angle $\beta_{1}(\alpha / 2)$ of the arch rib and $\beta(\alpha / 2)$ of whole cross section of the arch, and $m$ and $n$. For the case of support type A (Fig. 3), it is noticed that the influence of $n$ on $\beta_{1}(\alpha / 2)$ is not regular like that on $\beta(\alpha / 2)$ listed up in Table 1 . From Fig. 5 (showing the torsional deformation curve), it can be seen that the effect of $n$ on the curve $\beta(\alpha / 2)$ for the case of support type $\mathrm{A}$ is

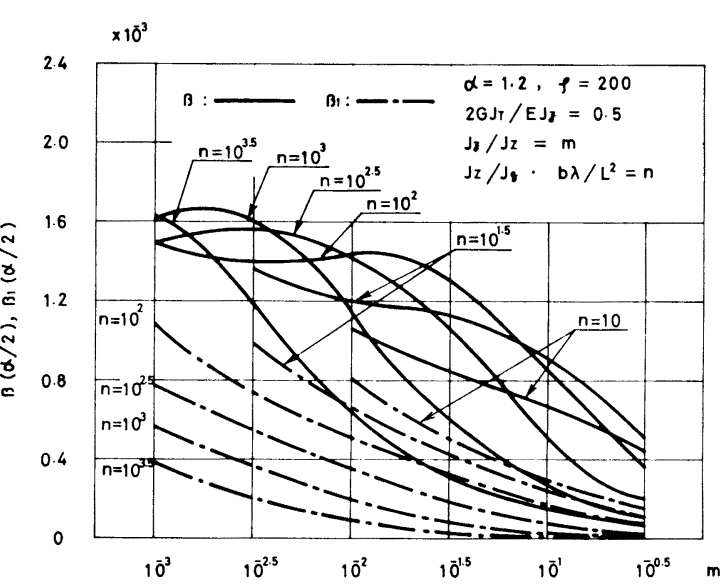

Fig. 3 Effect of $m, n$ on $\beta(\alpha / 2), \beta_{1}(\alpha / 2)$.

considerably complicated. However, it should be noted that for larger $n$, maximum values of $\beta$ 
occur rather at near points by the end supports than at the crown. In Fig. $4, \beta_{0}$ shows the twist angle of an equivalent circular beam at the $\alpha / 2 \mathrm{pt}$.. In Fig. 4 (support type B), for the cases $(l=0.5$, $\left.n=10, m<10^{-2.5}\right)$ and $\left(l=0.5, n=10^{1.5}, m<10^{-3}\right)$,

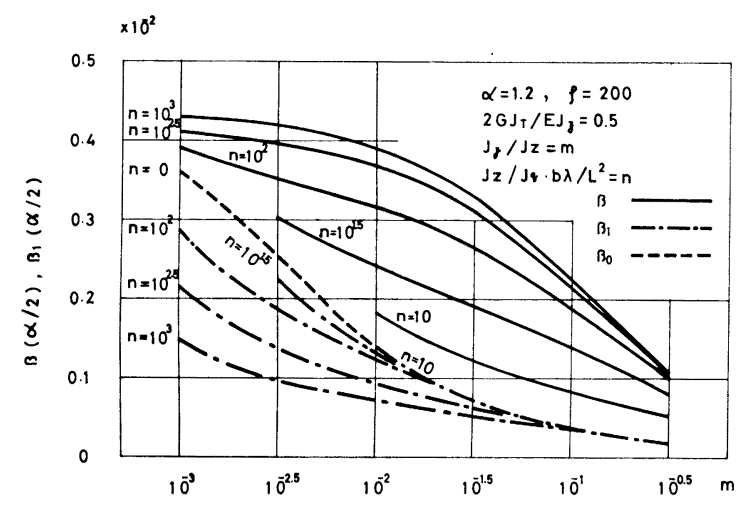

Fig. 4 Effect of $m, n$ on $\beta(\alpha / 2), \beta_{1}(\alpha / 2)$. (support type A) (support type B)

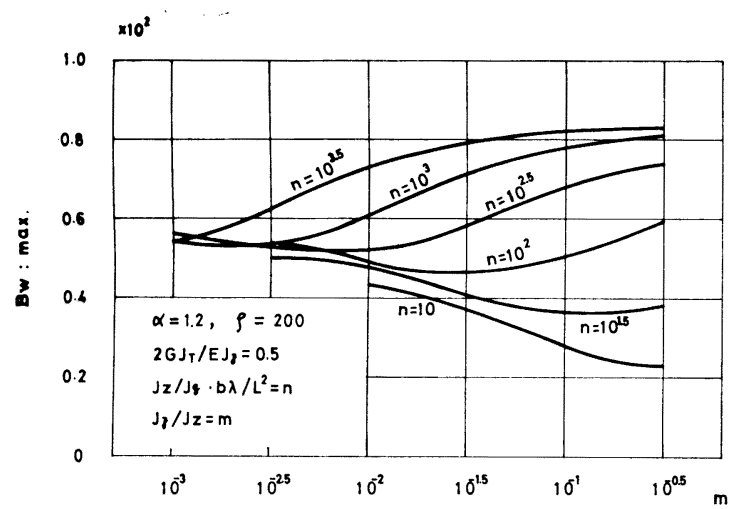

Fig. 5 Effect of $m, n$ on maximum value of $B_{W}$. (support type A)

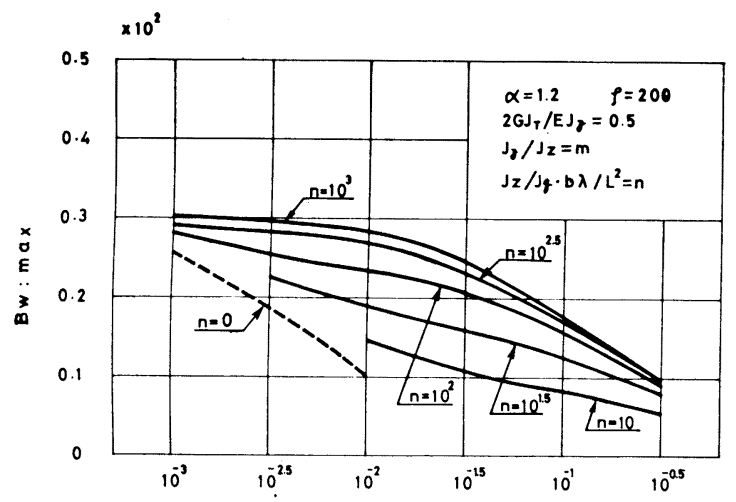

Fig. 6 Effect of $m, n$ on maximum value of $B_{W}$. (support type B) it is impossible to calculate numerically by reason of the aforesaid ill-conditioning of coefficient's matrix. This conditioning may due to that the deformation of the cross-sectional shape of the arch hardly occurs in the above mentioned cases. So that, in this case, it is suggested to analyse the arch as an equivalent circular beam with a rigid cross sectional shape. The lateral displacement shows nearly the same tendency with the twist angle of a whole cross section of the arch, except that its maximum value is produced always at the crown of the arch, but its illustration is omitted here. The relationship of the maximum warping normal stresses occured at the end cross section to the parameters $m$ and $n$ is illustrated for the case of support type $A$ in Fig. 5 . The maximum values of the warping stresses for the case of support type $B$ as shown in Fig. 6 occur in the neighborhood of the $\alpha \cdot 1 / 8$ and $\alpha \cdot 7 / 8$ pt. of the span and give nearly the same tendency with the twist angle of a whole cross section of the arch for that case as shown in Fig. 4. The warping stresses for support type $A$ are about twice as large as those of type $B$, if the parameters lie in the range of $\left(m=10^{-3}, n=10^{2} \sim 10^{3}\right)$ or $\left(m=10^{-0.5}, n=10\right)$. However, for the case ( $m$ $\left.=10^{-0.5}, n=10^{3}\right)$, it becomes about octuple.

b) Effect of Rigidity Ratio $l$

Figs. 7 and 8 show the effect of rigidity ratios $l$ and $m$ on the torsional deformation of the arch.

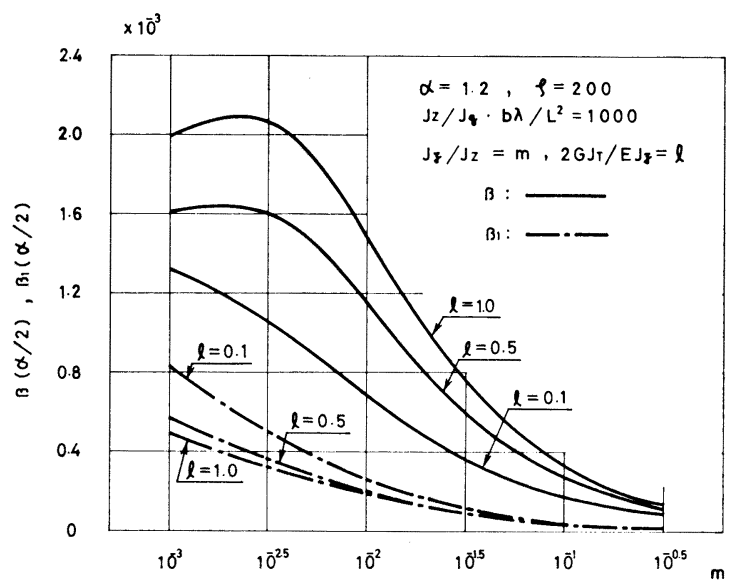

Fig. 7 Effect of $l, m$ on $\beta(\alpha / 2), \beta_{1}(\alpha / 2)$. (support type A)

It should be noticed that the effect of the torsional rigidity of the arch rib on the twist angle of a whole cross section of the arch appears more clearly for the case of the support type A (Fig. 7) than that of B (Fig. 8). This results indicate 
clearly the effect of the difference of end-supporting conditions.

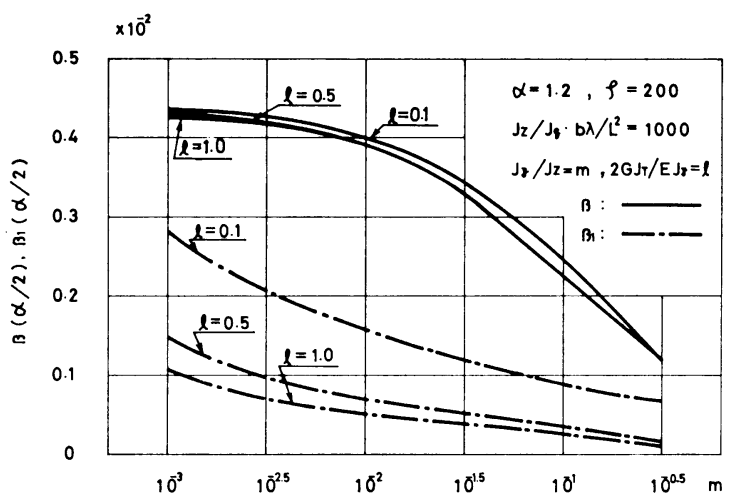

Fig. 8 Effect of $l, m$ on $\beta(\alpha / 2), \beta_{1}(\alpha / 2)$. (support type B)

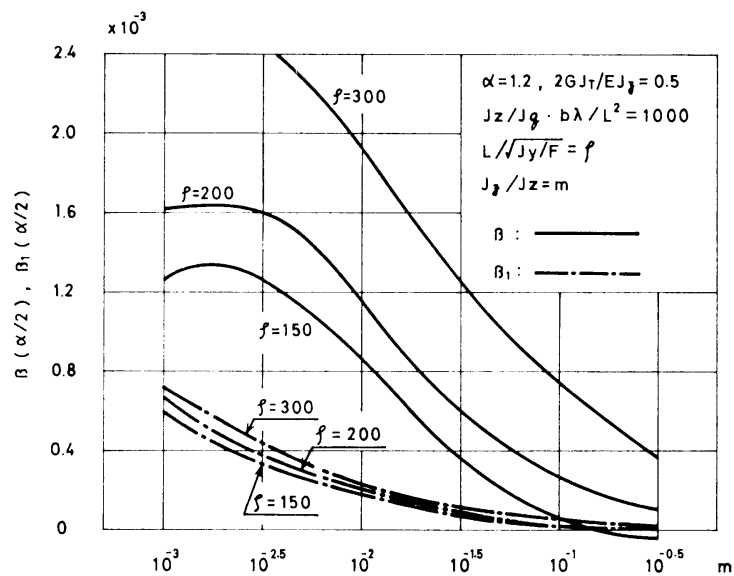

Fig. 9 Effect of $m, \rho$ on $\beta(\alpha / 2), \beta_{1}(\alpha / 2)$. (support type A)

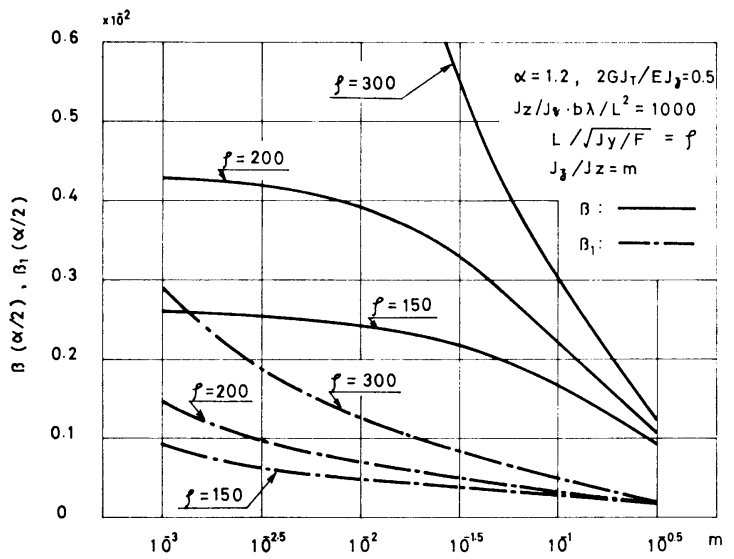

Fig. 10 Effect of $m, \rho$ on $\beta(\alpha / 2), \beta_{1}(\alpha / 2)$. (support type B) c) Effect of Slenderness Ratio $\rho$

Figs. 9 and 10 show the relationship of the flexural rigidity of arch ribs about the horizontal axis expressed as slenderness ratio $\rho$, to the torsional deformation of the arch. From Fig. 9 (support type A), for the case $m=10^{-2}$, the ratio of $\beta_{1}(\alpha / 2)$ for $\rho=300$ to that for $\rho=150$ is 0.75 and that of $\beta(\alpha / 2)$ is 0.45 . On the other hand, from Fig. 10 (support type B), the ratio of $\beta_{1}(\alpha / 2)$ and that of $\beta(\alpha / 2)$ are 0.37 and 0.33 respectively. The effect of $\rho$ on the torsional deformation appears more clearly for the case of support type $B$ than that of type A, furthermore, more clearly for $\beta(\alpha / 2)$ than for $\beta_{1}(\alpha / 2)$.

d) Effect of Central Angle $\alpha$, in Relation to $m$ and $n$

Fig. 11 shows the relationship of the twist angle ratio $\beta_{1}(\alpha / 2) / \beta(\alpha / 2)$ to the central angle $\alpha$ and the rigidity ratio $m$. For the case of an equivalent circular beam, the twist angle ration is 1.0. The case of support type A takes a different tendency with that of type $\mathrm{B}$, when $\alpha>1.2$ and $m<10^{-2}$; when $\alpha>1.2$, the results for support type A give the reverse tendency of type B. Fig. 12 shows the effect of $n$ and $\alpha$ on the twist angle ratio, and from this figure the effect of $\alpha$ cannot be seen practically when $\alpha>1.2$ for the case of support type A. Figs. 13 and 14 show the interaction of $\alpha, m$ and $n$ on the end moments. The effect of $m$ on the end-bending moment becomes insignificant when $\alpha>1.4$ for the case of support

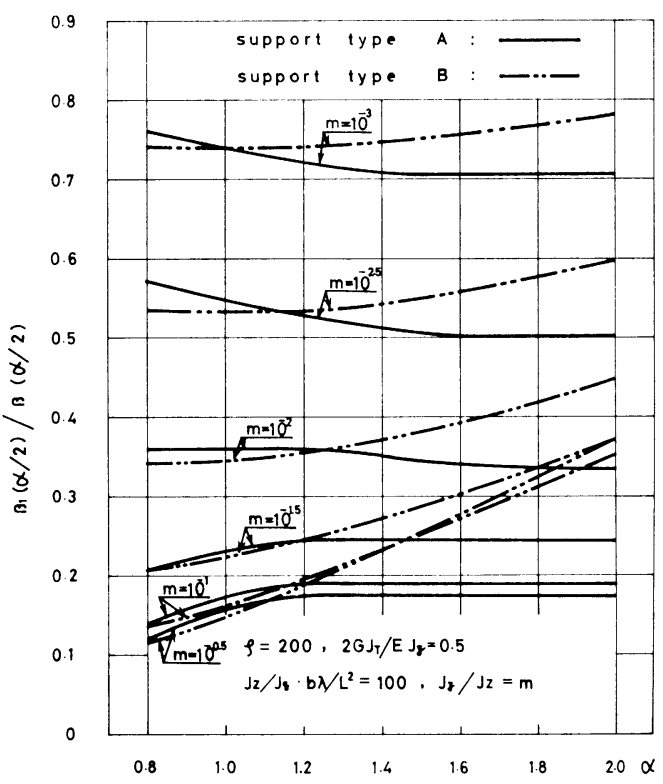

Fig. 11 Effect of $\alpha, m$ on $\beta_{1}(\alpha / 2) / \beta(\alpha / 2)$. 


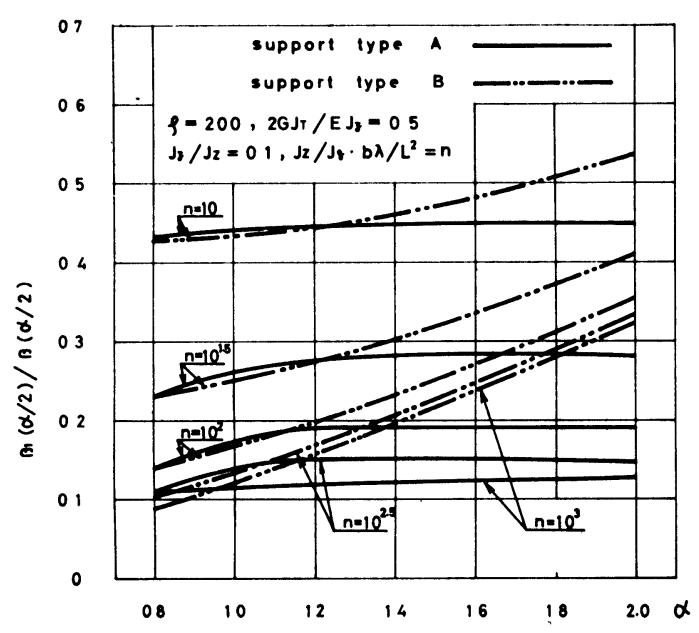

Fig. 12 Effect of $\alpha, n$ on $\beta_{1}(\alpha / 2) / \beta(\alpha / 2)$.

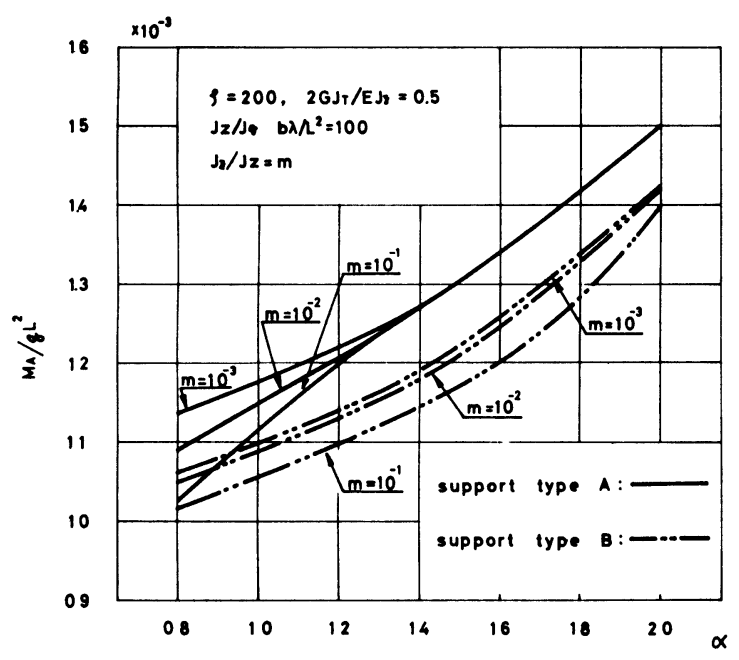

Fig. 13 Effect of $\alpha, m$ on $M_{A} / q L^{2}$.

type $A$ as shown in Fig. 13. However, on the end-twisting moment, $n$ becomes insignificant parameter when $\alpha>1.6$ for the case of type $B$ as shown in Fig. 14.

\section{CONCLUSION}

In this paper, the effect of biaxial flexural rigidity of arch ribs on the out-of-plane behavior of the arch are presented in relation to the interaction of flexural rigidity of cross beams and of torsional rigidity of ribs. The effect of change of the end-supporting conditions is also presented. These conditions taken up here are support type

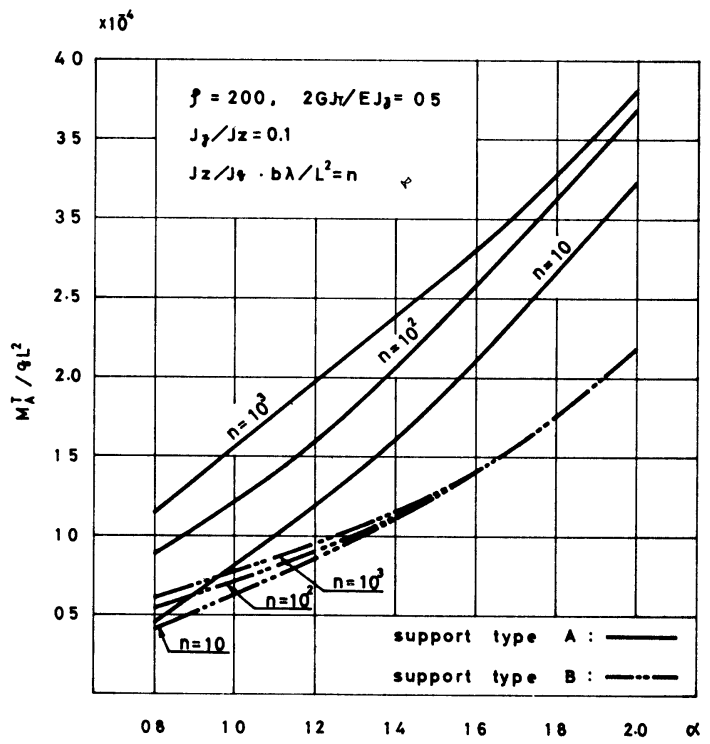

Fig. 14 Effect of $\alpha, n$ on $M_{A}^{T} / q L^{2}$.

$A$ and B, mathematically shown in Eqs. (4-1) and (4-2) respectively. The results of the analysis presented in this paper are discussed by numerical comparison with that of an equivalent circular beam.

The following conclusion can be described from the results presented in this paper.

1) Even if an arch has cross beams with low flexural rigidity, the arch having ribs with high flexural and torsional rigidity can be expected to have considerable out-of-plane resistance for the lateral deformation, according to the flxural rigidity of ribs.

2) The lateral deformation of arch for the case of support type $A$ is smaller than that of type $B$, however, the stresses of arch ribs for the case of type $A$ are larger. Especially for the arch having cross beams with low flexural rigidity and support type $A$, high stresses are produced near the end supports.

3) The out-of-plane behavior of an arch approaches that of an equivalent circular beam, as the flexural rigidity of arch ribs about the vertical radial axis decreases, and that of cross beams increases.

4) When the rigidity ratio $l$ and the slenderness ratio $\rho$ are large, arches should be analysed by the method presented in this paper.

5) For the case of support type B, as the central angle of arches increases, it may be reasonable to model the arches by an equivalent circular 


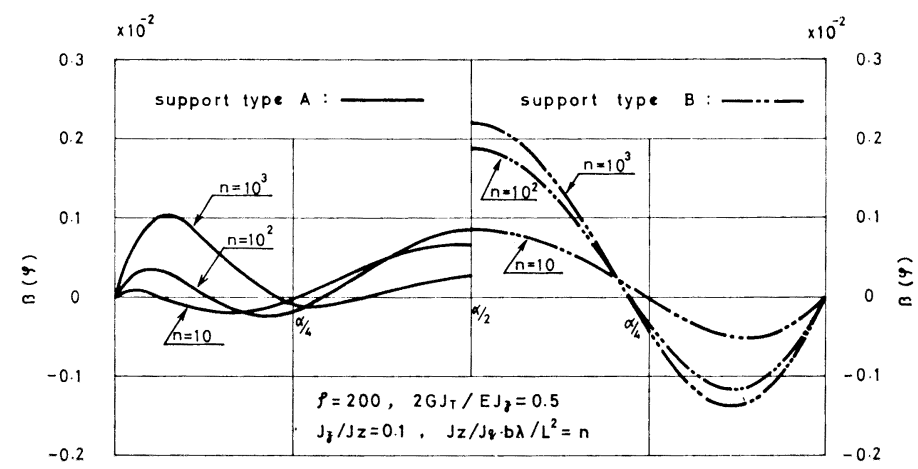

Fig. 15 Effect of $n$ on $\beta-\phi$ curve.

beams, however, for the case of type A, it is not applicable.

In this calculation, only a uniformly distributed load is taken up. By using the analysis method presented here for a concentrated load, the lateral stiffness of the arch can be obtained easily.

The computations were performed by using the NEAC-700 Computer of Tohoku University.

\section{APPENDIX I-COEFFICIENTS IN EQS. $(5-2 . a) \sim(5-2 . c)$}

The following values and functions are denoted in order to express coefficients of the initial constants of the external forces $\left(\bar{M}_{A}, \bar{M}_{B}, \bar{P}, \bar{Q}\right)$ in Eqs. (5-2.a) (5-2.c). The values are

$$
\begin{aligned}
& P_{1}=\frac{J_{z}}{J_{z}}, \quad P_{2}=\frac{G J_{T}}{E J_{q}} \frac{b \lambda}{6 R^{2}}, \quad P_{3}=\frac{J_{y}}{J_{Z}} \frac{b^{2}}{2 R^{2}}, \quad P=\frac{2 G J_{T}}{E J_{Z}} \\
& A_{1}=\frac{1}{P_{2}}+\frac{P_{1}}{P_{3}\left(1+P_{1}\right)}, \quad A_{2}=\frac{P_{4}}{P_{2} P_{3}}+\frac{P_{1}}{P_{3}\left(1+P_{1}\right)} \\
& A_{3}=\frac{1}{1+P_{1}}, \quad A_{4}=\frac{P_{1}}{1+P_{1}}, \quad A_{5}=A_{4}-A_{1} \\
& A_{6}=\frac{1}{P_{2}\left(1+P_{1}\right)}+\frac{1}{P_{3}\left(1+P_{1}\right)}+\frac{P_{1}}{P_{4}\left(1+P_{1}\right)} \\
& A_{7}=\frac{1}{P_{2} P_{3}}+\frac{P_{4}}{P_{2} P_{3}\left(1+P_{1}\right)}+\frac{P_{1}}{P_{3} P_{4}\left(1+P_{1}\right)} \\
& A_{8}=A_{1}+A_{3}, \quad A_{9}=\frac{1}{1+P_{1}}\left(1+\frac{P_{1}}{P_{4}}\right) \\
& A_{10}=\frac{1}{P_{2}\left(1+P_{1}\right)}, \quad A_{11}=A_{7}+\frac{1}{P_{3}\left(1+P_{1}\right)} \\
& A_{12}=\left(A_{3}\right)^{2}+A_{4} A_{9}, \quad A_{13}=A_{3} A_{6}+A_{4} A_{10} \\
& A_{14}=A_{3} A_{7}+A_{4} A_{11}
\end{aligned}
$$

and the functions are

$$
B_{1}{ }^{i}=\frac{X_{i}-Y_{i}+Z_{i}}{\left(a^{2}+1\right)\left(b^{2}+1\right)}
$$

$$
\begin{aligned}
& B_{2}^{i}=\frac{X_{i}+a^{2} Y_{i}+a^{4} Z_{i}}{\left(a^{2}-b^{2}\right)\left(a^{2}+1\right)} \\
& B_{3}{ }^{i}=\frac{X_{i}+b^{2} Y_{i}+b^{4} Z_{i}}{\left(b^{2}-a^{2}\right)\left(b^{2}+1\right)} \\
& C_{1}{ }^{i}=\frac{X_{i}-Y_{i}+Z_{i}}{\left(a^{2}+b^{2}-1\right)^{2}+4 a} \\
& C_{2}{ }^{i}=\frac{-\left(3 a^{2}-b^{2}+1\right) X_{i}-\left(a^{2}+b^{2}\right)\left(a^{2}+b^{2}-1\right) Y_{i}}{4 a\left(a^{2}+b^{2}\right)} \\
& \times \frac{+\left(a^{2}+b^{2}\right)\left\{\left(a^{2}+b^{2}\right)\left(a^{2}+b^{2}-1\right)+4 a\right\} Z_{i}}{\left\{\left(a^{2}+b^{2}-1\right)^{2}+4 a\right\}} \\
& C_{3}{ }^{i}=\frac{\left(2 a^{2}-2 b^{2}+1\right) X_{i}+\left(a^{2}+b^{2}\right)^{2}\left(Y_{i}-Z_{i}\right)}{2\left(a^{2}+b^{2}\right)\left\{\left(a^{2}+b^{2}-1\right)^{2}+4 a\right\}}
\end{aligned}
$$

Let us calculate the values listed in Table 3,4 by using Eqs. (7-1), (7-2.a) (7-2.c) and (7-3.a) (7-3.c). By substituting the values listed in Table 3,4 into Table 5 and 6 , the coefficients in Eqs. (5-2.b) and (5-2.c) can be obtained, respectively.

The same calculating procedure with the coefficients in Eq. (5-2.b) can be applied for those in Eq. (5-2.a), using $\left(X_{i}, Y_{i}, Z_{i}\right)$ listed in Table 3 instead of those in Table 2. The coefficients take a different form according to the range of $\left(A_{1}\right)^{2}-4 A_{3}$. The case of $\left(A_{1}\right)^{2}-4 A_{3}=0$ can be obtained similarly, but it is omitted here.

Table 2

\begin{tabular}{c|c|c|c|c}
\hline & $i=1$ & $i=2$ & $i=3$ & $i=4$ \\
\hline$X_{i}$ & 0 & $A_{2}$ & $-A_{8}$ & $-A_{11}$ \\
\hline$Y_{i}$ & $-A_{8}$ & $A_{3}$ & 0 & $A_{10}$ \\
\hline$Z_{i}$ & 0 & 1 & 0 & $A_{9}$ \\
\hline
\end{tabular}


Table 3

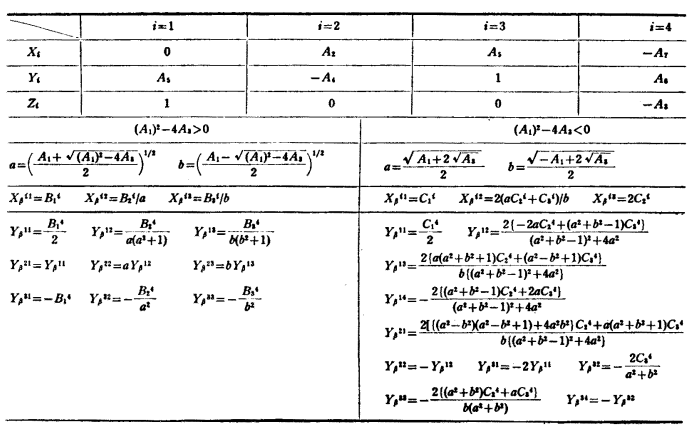

Table 4

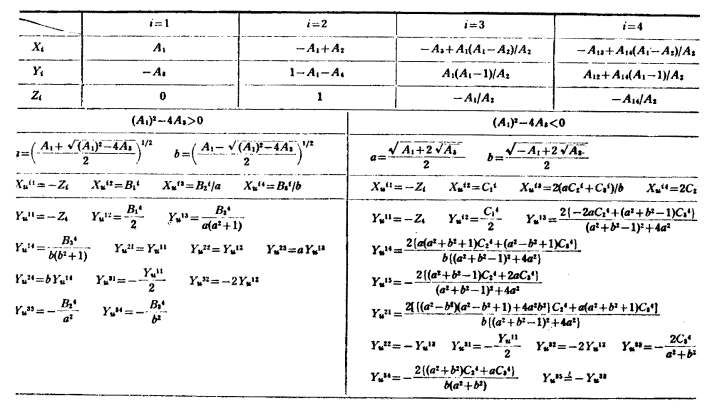

Table 5

\begin{tabular}{|c|c|c|}
\hline & $\left(A_{1}\right)^{2}-4 A_{3}>0$ & $\left(A_{1}\right)^{2}-4 A_{3}<0$ \\
\hline$a b$ & $a=\left(\frac{A_{1}+\sqrt{\left(A_{1}\right)^{2}}}{2}=4 A_{3}\right)^{1 / 2} \quad b=\left(\frac{A_{1}-\sqrt{\left(A_{1}\right)^{2}-4 A_{2}}}{2}\right)^{1 / 2}$ & $a=\frac{\sqrt{A_{1}+2 \sqrt{A_{3}}}}{2} \quad b=\frac{\sqrt{-A_{1}+2 \sqrt{A_{s}^{-}}}}{2}$ \\
\hline \multirow[t]{2}{*}{$X_{\dot{p}}^{\prime}(\phi)$} & $X_{\beta}^{i 1} \sin \phi+X_{\beta}^{i z} \sinh a \phi+X_{\beta}^{i 3} \sinh b \phi$ & $X_{\beta}^{41} \sin \phi+X_{\beta}^{i 2} \cosh a \phi \cdot \sin b \phi+X_{\phi}^{i 3} \sinh a \phi \cdot \cos b \phi$ \\
\hline & $X_{\beta}^{z}(\phi)=X_{\beta}^{21} \cos \phi+X_{\beta}^{2 z} \cosh a \phi+X_{\beta}^{2 s} \cosh b \phi$ & $X_{\beta^{2}}^{2}(\dot{\phi})=X_{\beta^{21}} \cos \phi+X_{\beta^{2}}^{22} \sinh a \phi \cdot \sin b \phi+X_{\beta^{23}} \cosh a \phi \cdot \cos b \phi$ \\
\hline$Y_{\beta}^{1}(\phi)$ & $\begin{array}{l}\cos \alpha \\
\sin \alpha \\
Y_{\beta}{ }^{31}(1-\cos \phi)-Y_{\beta}{ }^{22}(\cosh a \phi-1)-Y_{\beta}{ }^{33}(\cosh b \phi-1)\end{array}$ & $\begin{array}{l}\frac{\cos \alpha}{\sin \alpha}\left[Y_{\beta}^{11}(\sin \phi-\phi \cos \phi)+Y_{\beta}^{12} \sin \phi+Y_{\beta}^{13} \cosh a \phi \cdot \sin b \phi+\right. \\
\left.Y_{\beta^{1}} \sinh a \phi \cdot \cos b \phi\right]-Y_{\beta}^{31}(1-\cos \phi)-Y_{\beta}^{32} \sinh a \phi-Y_{\beta}^{34} \cosh a \phi \cdot \cos b \phi\end{array}$ \\
\hline$Y_{\beta}^{2}(\phi)$ & $-\frac{1}{\sin \alpha}\left[Y_{\beta}^{11}(\sin \phi-\phi \cos \phi)+Y_{\beta}^{12}(\sinh a \phi-a \sin \phi)+Y_{\beta}^{13}(\sinh b \phi-b \cos \phi)\right]$ & $-\frac{1}{\sin \alpha}\left[Y_{\beta}^{11}(\sin \phi-\phi \cos \phi)+Y_{\beta}^{12} \sin \phi+Y_{\beta}^{13} \cosh a \phi \cdot \sin b \phi+Y_{\beta}^{14} \sin b a \phi \cdot \cos b \phi\right]$ \\
\hline$Y_{\beta}{ }^{3}(\phi)$ & 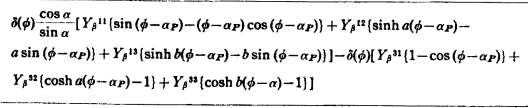 & $\begin{array}{l}\delta(\phi) \frac{\cos \alpha}{\sin \alpha}\left\{Y_{\beta}^{11}\left(\sin \left(\phi-\alpha_{P}\right)-\left(\phi-\alpha_{P}\right) \cos \left(\phi-\alpha_{P}\right)\right]+Y_{\beta}^{12} \sin \left(\phi-\alpha_{P}\right)+\right. \\
\left.\left.Y_{\beta}^{13} \cosh a\left(\phi-\alpha_{P}\right) \cdot \sin b\left(\phi-\alpha_{P}\right)+Y_{\beta}^{14} \sinh \alpha\left(\phi-\alpha_{P}\right) \cdot \cos \phi \phi-\alpha_{P}\right)\right]- \\
\hat{\delta}_{2}(\phi)\left[Y_{\beta^{31}}\left(1-\cos \left(\phi-\alpha_{P}\right)\right\}+Y_{\beta}^{32} \sinh a\left(\phi-\alpha_{P}\right)+Y_{\beta}^{34} \cosh \alpha\left(\phi-\alpha_{P}\right) \cdot \cos b\left(\phi-\alpha_{P}\right)\right]\end{array}$ \\
\hline$Y_{\beta}^{*}(\phi)$ & 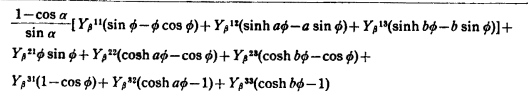 & $\begin{array}{l}\frac{1-\cos \alpha}{\sin \alpha}\left[Y_{\beta}^{11}(\sin \phi-\phi \cos \phi)+Y_{\beta}^{12} \sin \phi+Y_{\beta}^{13} \cosh a \phi \sin b \phi+\right. \\
\left.Y_{\beta}^{11} \sinh a \phi \cdot \cos b \phi\right]+Y_{\beta}^{11} \phi \sin \phi+Y_{\hat{\beta}}^{12} \cos \phi+Y_{\beta}^{21} \sinh a \phi \cdot \sin b \phi+ \\
Y_{\beta}^{22} \cosh a \phi \cdot \cos b \phi+Y_{\beta}^{31}(1-\cos \phi)+Y_{\beta}^{32}+Y_{\beta}^{33} \sinh a \phi \cdot \sin b \phi+Y_{\beta}^{31} \cosh a \phi \cdot \cos b \phi\end{array}$ \\
\hline
\end{tabular}

Table 6

\begin{tabular}{|c|c|c|}
\hline & $\left(A_{1}\right)^{2}-4 A_{9}>0$ & $\left(A_{1}\right)^{2}-4 A_{3}<0$ \\
\hline$a \quad b$ & $a=\left(\frac{A_{1}+\sqrt{\left(A_{1}\right)^{2}-4 A_{8}}}{2}\right)^{1 / 2} \quad b=\left(\frac{A_{1}-\sqrt{\left(A_{1}\right)^{2}-4 A_{8}}}{2}\right)^{1 / 2}$ & $a=\frac{\sqrt{A_{1}+2 \sqrt{A_{1}}}}{2} \quad b=\frac{\sqrt{-A_{1}+2 \sqrt{A_{1}}}}{2}$ \\
\hline \multirow[t]{2}{*}{$X_{u^{1}}(\phi)$} & $X_{u^{11}} \phi+X_{u^{12}} \sin \phi+X_{u^{14}} \sinh a \phi+X_{u^{4}} \sinh b \phi$ & $X_{u^{41}} \phi+X_{u}{ }^{42} \sin \phi+X_{u}{ }^{43} \cosh a \phi \cdot \sin b \phi+X_{u}{ }^{4} \sinh a \phi \cdot \cos b \phi$ \\
\hline & $\left.X_{u^{2}}{ }^{2} \phi\right)=X_{u^{21}}+X_{u^{2 z}} \cos \phi+X_{u^{23}} \cosh a \phi+X_{u^{2 z}} \cosh b \dot{\phi}$ & $X_{u^{2}}(\phi)=X_{u^{21}}+X_{u^{2}}^{z} \cos \phi+X_{u^{23}} \sinh a \phi \cdot \sin b \phi+X_{u^{24}} \cosh a \phi \cdot \cos b \phi$ \\
\hline$Y_{u^{1}}(\phi)$ & 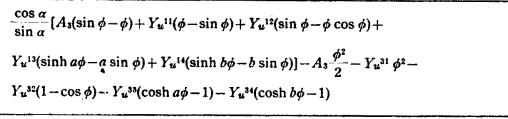 & 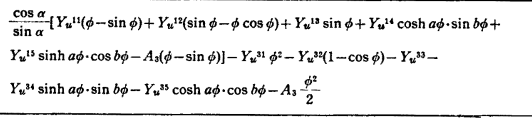 \\
\hline$Y_{u^{2}}(\phi)$ & $\begin{array}{l}-\frac{1}{\sin \alpha}\left[A_{v}(\sin \phi-\phi)+Y_{u}^{11}(\phi-\sin \phi)+Y_{u^{12}}(\sin \phi-\phi \cos \phi)+\right. \\
\left.Y_{u^{13}}(\sinh a \phi-a \sin \phi)+Y_{u^{14}}(\sinh b \phi-b \sin \phi)\right]\end{array}$ & $\begin{array}{l}-\frac{1}{\sin \alpha}\left[Y_{u^{11}}{ }^{11}(\phi-\sin \phi)+Y_{u^{12}}(\sin \phi-\phi \cos \phi)+Y_{u^{13}} \sin \phi+\right. \\
Y_{u^{14}} \cosh a \phi \cdot \sin b \phi+Y_{u}^{15} \sinh a \phi \cdot \cos b \phi-A_{3}(\phi-\sin \phi)\end{array}$ \\
\hline$Y_{u^{3}(\phi)}$ & 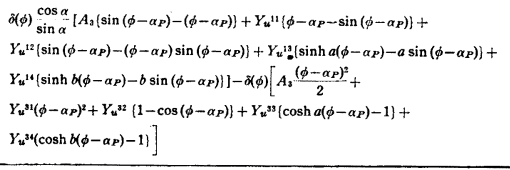 & 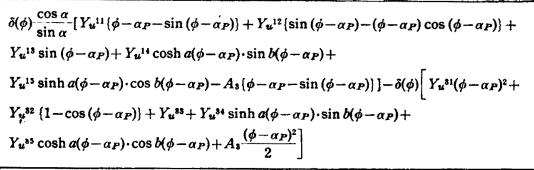 \\
\hline$Y_{u^{4}}(\phi)$ & 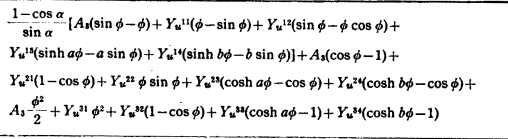 & 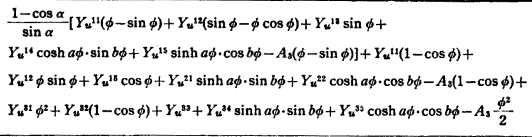 \\
\hline
\end{tabular}




\section{APPENDIX II-NOTATIONS}

The following symbols are used in this paper. $X, Y, Z$ : coordinate system

$u^{(\mathrm{I}),(\mathrm{II})}:$ displacement of shear center of arch rib in the $Y$-direction

$v^{(I),(I I)} \quad$ : displacement of shear center of arch rib in the $Z$-direction

$w^{(\mathrm{I}),(\mathrm{II})}$ : displacement of shear center of arch rib in the $X$-direction

$\beta^{(I),(I I)}, \beta_{1}$ : twist angle of arch rib

$u, v$ : displacement of a whole cross section of arch in the $Y$ - and $Z$-direction

$\beta \quad$ : twist angle of a whole cross section of arch

$E \quad$ : modulus of elasticity

$G \quad$ : shear modulus

$J_{z}, J_{y} \quad$ : moment of inertia of arch rib about $Z$ - and $Y$-axis

$J_{T} \quad$ : St. Venant torsion constant of arch rib

$J_{Z} \quad$ : moment of inertia of a whole cross section of arch

$R \quad$ : radius of curvature

$L \quad$ : span length of arch

$b \quad$ : distance between a center of a cross section of an arch rib and another one

$\phi \quad:$ angular coordinate

$M_{t} \quad$ : torsional moment acting on arch rib

$M_{z} \quad$ : bending moment about $Z$-axis acting on arch rib

$M_{y} \quad$ : bending moment about $Y$-axis acting on arch rib

$M_{T} \quad$ : torsional moment acting on arch

$M_{Z} \quad$ : bending moment about $Z$-axis acting on arch
$Q^{(1),(11)}:$ vertical shear force acting on arch rib

$J_{q} \quad:$ moment of inertia of cross beam

$M_{q} \quad$ : bending moment about $X$-axis acting on ends of cross beam

$m_{q} \quad:$ Eq. $(2-10)$

$Q \quad$ : transverse shear force acting on arch

$P \quad:$ concentrated load

$q \quad:$ uniformly distributed load

$\alpha \quad:$ central angle of arch

$\alpha_{P} \quad:$ angular coordinate pointing position of a concentrated load

$\delta(\phi) \quad$ : Eq. (3-3)

$M_{A}, M_{B}$ : end-bending moment

$M_{A}{ }^{T} \quad$ : end-twisting moment

$M_{1}, M_{2} \quad$ : Eqs. (3-1.a), (3-1.c)

$B_{W}(\phi) \quad$ : Eq. (4-3)

$\sigma_{W}(\phi) \quad:$ warping normal stress, Eq. (4-4)

$e \quad:$ distance between neutral axis and top or bottom surface of arch rib

$A \quad$ : cross sectional area of arch rib

$\lambda \quad:$ interval between cross beams

\section{REFERENCES}

1) Okamoto, S.：“軸線を含む面に垂直なる荷重を 受ける円形曲り梁の研究”, Jour. of J.S.C.E., No. 29, Vol. 3, 1943 (in Japanese).

2) Donald, P. and Godden, W.: “The Transverse Behaviour of Laterally Unsupported Parabolic Arches", The Structural Engineer, No. 6, Vol. 41, June, 1963.

3) Kuranishi, S.: "Analysis of Arch Bridge under Certain Lateral Forces", Trans. of J.S.C.E., No. 73, 1961 (in Japanese).

4) Chin Fung Kee: "The Design of the Unbraced Stabbogen Arch", The Structural Engineer, No. 9, Vol. 37, September, 1959. 